\title{
On the nonlinear dynamics of automated vehicles - a nonholonomic approach
}

\author{
Balázs Várszegi ${ }^{1, a, b}$, Dénes Takács ${ }^{\mathrm{a}}$, Gábor Orosz $^{\mathrm{c}}$ \\ ${ }^{a}$ Department of Applied Mechanics, Budapest University of Technology and Economics, Budapest, Hungary, \\ $H-1111$ \\ ${ }^{b}$ MTA-BME Lendlet Human Balancing Research Group, Budapest, Hungary, H-1111 \\ ${ }^{c}$ Department of Mechanical Engineering, University of Michigan, Ann Arbor, MI 48109, USA
}

\begin{abstract}
A simple mechanical model for the lateral and yaw motion of a vehicle is presented while taking into account rolling constraints. The governing equations are derived by utilizing the Appellian framework. Analytical and numerical bifurcation analysis is performed while utilizing a PD controller. The results provide insight into the local and global stability of forward and reverse motion of automated passenger vehicles and harvesters.
\end{abstract}

Keywords: nonholonomic mechanics, vehicle dynamics, steering control, stability

\section{Introduction}

Automated driving is one of the most significant step for road transportation since the construction of the first motor vehicle. It promises significant steps in safety, driving comfort and fuel economy. However, eliminating the human operator puts more stringent requirements for the performance of the steering controllers so that the planned vehicle trajectories can be executed even in the presence of external disturbances. In this paper, we utilize state-of-the-art analytical and numerical techniques in order to design controllers that may guarantee the performance of automated vehicles at the nonlinear level.

In practice, automated steering is typically achieved in a hierarchical manner. A higher-level controller is responsible for assigning the appropriate steering angle based on the information collected by sensors about the vehicle and its environment. This can be achieved via several different

\footnotetext{
${ }^{*}$ Corresponding author

Email addresses: varszegi@mm.bme.hu (Balázs Várszegi), takacs@mm.bme.hu (Dénes Takács), orosz@umich.edu (Gábor Orosz)
} 
control approaches. For example, one may use simple PID control [1], model predictive control [2, 3, 4, or other feedforward and feedback algorithms [5, 6]. In the mean time the lower level controller aims to match the steering angle to that assigned at the higher level through the steering mechanism. This latter problem is the focus of this study and we follow a model based approach.

Our goal is to study automated steering problems using analytical tools which requires simplified mechanical models. These models are typically of nonholonomic type due to the kinematic constraints describing rolling wheels; see [7, 2, 8, with tire models of different levels of complexity. While nonholonomic mechanics goes back more than 100 years [9], it mainly gained popularity the second half of the previous century [10, 11, 12. These types of models can be used to describe a wide variety of vehicles including sleighs [13], skateboards [14, 15], and bicycles [16, 17]. In this paper we develop nonholonomic models for automobiles and harvesters and investigate their nonlinear dynamics.

Most studies in automotive steering control focus on linear investigations around a stationary motion. However, studying the nonlinear dynamics of vehicles is necessary to understand a global picture. For example, the appearance of a wobbling motion after a large enough perturbation may lead to accidents despite the fact that the stationary motion is linearly stable. The effects of nonlinearities on wheel dynamics have been studied in [18, 19, 20, 21, while other studies focused on the nonlinear analyses of more complex vehicle and driver models [22, 23, 24, 25, 26]. However, none of these contributions contains information on the effect of the trail of the steered wheels nor presents bifurcation diagrams in terms of the essential vehicle parameters (e.g. inertial parameters, longitudinal speed, trail, control gains).

Having such analysis is important to ensure the safety and reliability of automated vehicles. Therefore, in this paper we use a simplified mechanical model with rigid wheels while taking into account the trail of the steered wheels and we utilize a simple control law to adjust the steering angle. We draw bifurcation diagrams to analyze the effects of the speed and the trail on the nonlinear behavior for different inertial and control parameter setups. With the help of this global bifurcation analysis we characterize how the identified dynamical phenomena manifest themselves on automated automobiles and harvesters. This allows us to draw conclusions about the steering behavior in different forward and reverse speed ranges.

The contents of the article are organized as follows. Section 2 introduces the mechanical model and the detailed derivation of the equations of motion. In Section 3 , the mathematical analysis 
of the equations is presented with special attention to the stability and bifurcations of equilibria and singularities. In particular, pitchfork and Hopf bifurcations are characterized using analytical tools. Section 4 presents the interpretation of the results to different types of vehicles. Section 5 concludes the results and lays out some future research directions.

\section{Modelling the dynamics of automated vehicles}

In this section we derive the mathematical model used to analyze the lateral and yaw dynamics of the automated vehicle. We consider rigid wheels with point contacts which result in a low-

\subsection{Mechanical model}

We consider the so-called bicycle model or two-wheeled vehicle model (see in Fig. 1), which assumes that the vehicle can only move in the horizontal plane (i.e., vertical dynamics is neglected). The vehicle consists of two main parts: the chassis (including the rear wheel) and the steering system (the front wheel with caster). For simplicity the masses of the wheels are omitted (see more details below) so the vehicle is represented as two rigid bodies. The inertial and geometrical parameters of the chassis are the following: $m_{\mathrm{ch}}$ denotes the mass of the chassis, $J_{\mathrm{ch}}$ represent its mass moment of inertia about the center of mass $\mathrm{C}, l$ is the distance between points $\mathrm{R}$ and $\mathrm{F}$, and $b_{\mathrm{ch}}$ is the distance between points $\mathrm{R}$ and $\mathrm{C}$. The parameters belonging to the steering system are: $m_{\mathrm{st}}$ is the mass of the steering system, $J_{\mathrm{st}}$ is its mass moment of inertia about the center of mass $\mathrm{S}, b_{\mathrm{st}}$ gives the distance between the points $\mathrm{F}$ and $\mathrm{S}$, while $e$ represents the caster length, i.e., the distance between the center of the front wheel $\mathrm{W}$ and the steering hinge $\mathrm{F}$. Note that, the wheelbase is given by $l-e$ which is the distance between the wheel center points $\mathrm{R}$ and $\mathrm{W}$ in case of an unsteered vehicle.

In order to unambiguously describe the configuration of two rigid bodies connected by a hinge in the horizontal plane we need four configurational coordinates. Here we chose the position of the rear point $\mathrm{R}$, that is, $x \equiv X_{\mathrm{R}}$ and $y \equiv Y_{\mathrm{R}}$, the yaw angle $\psi$, and the steering angle $\gamma$. We assume point contacts between the rigid wheels and the ground and assume that the wheels are rolling without slipping. This results in kinematic constrains: the directions of the velocities of the centers of the wheels are aligned with the directions of the wheels, i.e., the slip angles are zero; see wheel rotates with a constant angular velocity which implies that the longitudinal speed $v:=\left|\mathbf{v}_{\mathrm{R}}\right|$ 


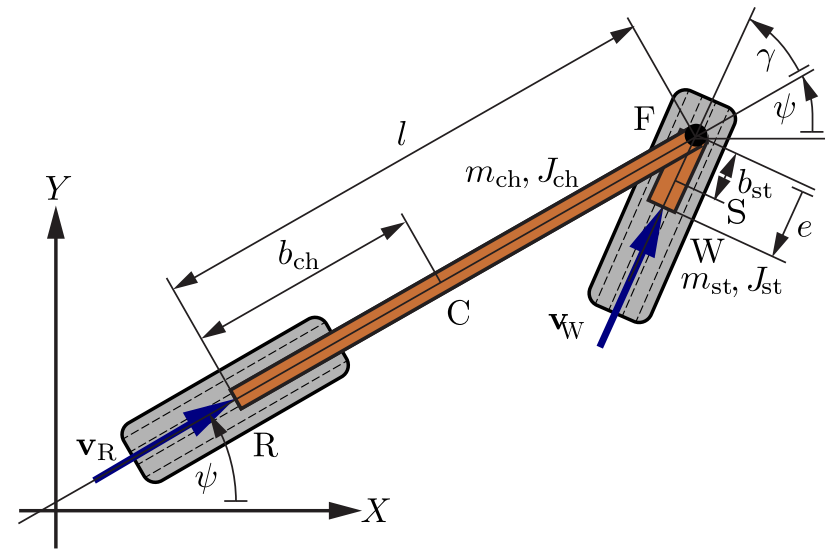

Figure 1: The mechanical model used in this paper.

is constant. Note that below we will use the convention $v>0$ when $\mathbf{v}_{\mathrm{R}}$ points toward $\mathrm{F}$ and $v<0$ when it points to the opposite direction.

Fig. 2 highlights four different application of the mechanical model for vehicles. Panels (a) and (b) corresponds to positive caster length $e>0$ and they allow us to investigate the forward and reverse motion of automobiles. Moreover, the case $v>0$ shown in panel (a) can also be used to describe the forward motion of American shopping carts (with fixed rear wheels and swivelling front wheels). On the other hand, panels (c) and (d) corresponds to negative caster length $e<0$ and these can be used to model the forward and reverse motion of harvesters, and the case $v<0$ shown in panel (c) also describes the reverse motion of the shopping carts.

\subsection{Mathematical model}

In order to turn the constructed mechanical model into a set of differential equations we apply some tools from analytical mechanics [27, 28, 10, 29. Due to the kinematic constraints of rolling the system is nonholonomic [13]. In this case the Lagrangian approach results in a set of algebraic differential equations containing Lagrange multipliers corresponding to the kinematic constrains. To obtain differential equations one must eliminate these multipliers which typically requires tedious algebraic manipulations. Instead we use the Appellian approach that directly provides a set of differential equations. Rather than simply using the derivatives of the configurational coordinates, we use pseudo velocities $\sigma_{i}$. These are chosen intuitively, but must satisfy the kinematic constraints. 
(a)

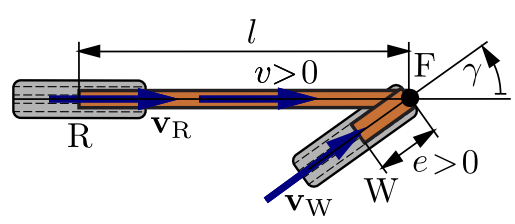

(b)

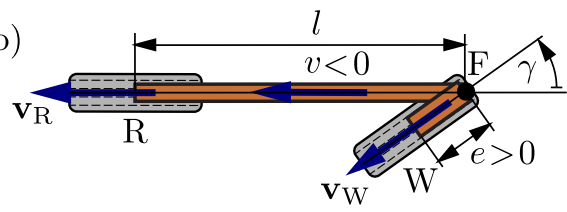

(c)

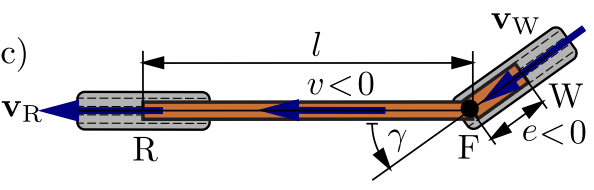

(d)

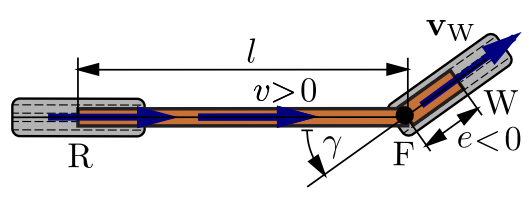

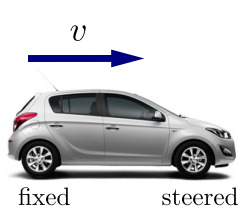
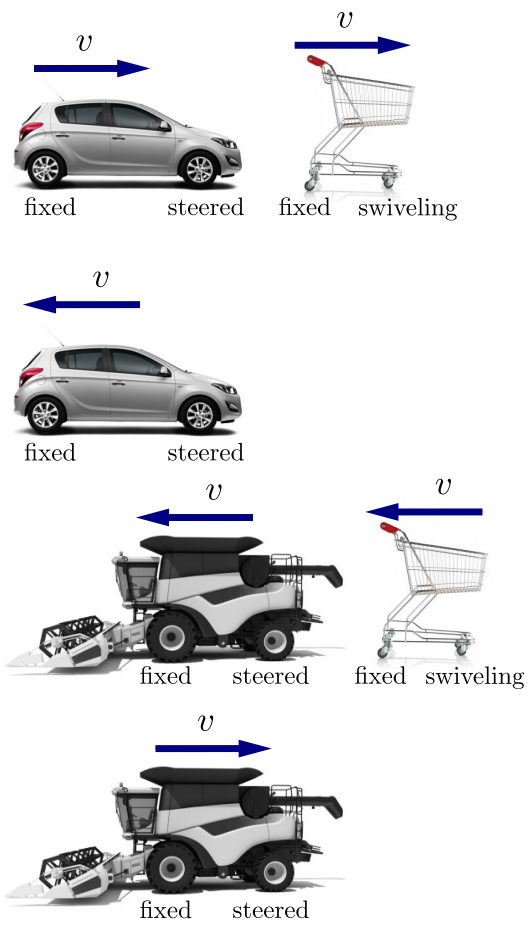

Figure 2: The possible setups of the model and the corresponding practical examples; setup (a) with $v>0, e>0$ corresponds to the forward motion of automobiles and pushed shopping carts; setup (b) with $v<0, e>0$ corresponds to the reverse motion of cars; setup (c) with $v<0, e<0$ corresponds to the forward motion of harvesters and pulled shopping carts; setup (d) with $v>0, e<0$ corresponds to the reverse motion of harvesters.

Then, we construct the acceleration energy $\mathcal{A}$ to obtain Appell-Gibbs equations in the form

$$
\frac{\partial \mathcal{A}}{\partial \dot{\sigma}_{i}}=\Gamma_{i}
$$

where $\dot{\sigma}_{i}$ is the pseudo acceleration and $\Gamma_{i}$ denotes the pseudo force. These equations shall be augmented by the kinematic constraints to form a closed system.

For the bicycle model described above the kinematic constraints describe that the directions of the velocities of the wheel center points $\mathrm{R}$ and $\mathrm{W}$ are aligned with the directions of the corresponding wheels:

$$
\begin{aligned}
\dot{x} \sin \psi-\dot{y} \cos \psi & =0, \\
\dot{x} \sin (\gamma+\psi)-\dot{y} \cos (\gamma+\psi)+(e-l \cos \gamma) \dot{\psi}+e \dot{\gamma} & =0,
\end{aligned}
$$


while the prescribed longitudinal velocity is expressed by

$$
\dot{x} \cos \psi+\dot{y} \sin \psi=v .
$$

As we have four generalized coordinates and three kinematic constraint, only one pseudo velocity is required. Here, we choose the relative angular speed of the caster-wheel system, i.e.,

$$
\sigma:=\dot{\gamma}
$$

Using the constraining equations (2), (3), (4) and definition of the pseudo velocity (5), the generalized velocities can be expressed as

$$
\left[\begin{array}{c}
\dot{x} \\
\dot{y} \\
\dot{\psi} \\
\dot{\gamma}
\end{array}\right]=\left[\begin{array}{c}
v \cos \psi \\
v \sin \psi \\
\frac{e \sigma+v \sin \gamma}{l \cos \gamma-e} \\
\sigma
\end{array}\right]
$$

while the corresponding accelerations can be obtained as

$$
\left[\begin{array}{c}
\ddot{x} \\
\ddot{y} \\
\ddot{\psi} \\
\ddot{\gamma}
\end{array}\right]=\left[\begin{array}{c}
-v \sin \psi \frac{e \sigma+v \sin \gamma}{l \cos \gamma-e} \\
v \cos \psi \frac{e \sigma+v \sin \gamma}{l \cos \gamma-e} \\
\frac{e \dot{\sigma}+\sigma v \cos \gamma}{l \cos \gamma-e}+\frac{l \sigma \sin \gamma(e \sigma+v \sin \gamma)}{(l \cos \gamma-e)^{2}} \\
\dot{\sigma}
\end{array}\right] .
$$

Considering the two rigid bodies, representing the chassis and the steering system, the acceleration energy can be constructed as

$$
\mathcal{A}=\frac{1}{2} m a_{\mathrm{C}}^{2}+\frac{1}{2} J_{\mathrm{ch}} \alpha_{\mathrm{ch}}^{2}+\frac{1}{2} m_{\mathrm{st}} a_{\mathrm{S}}^{2}+\frac{1}{2} J_{\mathrm{st}} \alpha_{\mathrm{st}}^{2}+\ldots,
$$

where the terms which do not contain $\dot{\sigma}$ are not spelled out. To simplify the matter the mass of the steering system is neglected, that is, $m_{\mathrm{st}} \approx 0$. The acceleration of the center of mass of the chassis is given by

$$
\mathbf{a}_{\mathrm{C}}=\left[\begin{array}{c}
\ddot{x}-b_{\mathrm{ch}}\left(\dot{\psi}^{2} \cos \psi+\ddot{\psi} \sin \psi\right) \\
\ddot{y}+b_{\mathrm{ch}}\left(\ddot{\psi} \cos \psi-\dot{\psi}^{2} \sin \psi\right)
\end{array}\right],
$$

while

$$
\alpha_{\mathrm{ch}}=\ddot{\psi} \quad \text { and } \quad \alpha_{\mathrm{st}}=\ddot{\psi}+\ddot{\gamma}
$$

are the angular acceleration of the chassis and the steering system, respectively. 
Using the generalized velocities (6) and accelerations (7), the acceleration energy can be written as

$$
\begin{aligned}
\frac{1}{J_{\mathrm{st}}} \mathcal{A}= & \frac{E^{2} \theta_{2}+\cos ^{2} \gamma}{2(E-\cos \gamma)^{2}} \dot{\sigma}^{2} \\
- & \left(E \frac{\left(E \theta_{2}+\cos \gamma\right) \sin \gamma}{(E-\cos \gamma)^{3}} \sigma^{2}-V E \frac{E^{2} \theta_{1}-\theta_{2}+\cos ^{2} \gamma}{(E-\cos \gamma)^{3}} \sigma\right. \\
& \left.-V \frac{\left(E^{2}\left(\theta_{2}-\theta_{1}\right)-1\right) \cos \gamma}{(E-\cos \gamma)^{3}} \sigma-V^{2} \frac{E \theta_{1} \sin \gamma}{(E-\cos \gamma)^{2}}\right) \dot{\sigma}+\ldots
\end{aligned}
$$

where the rescaled speed $V$, the dimensionless caster length $E$, and the dimensionless mass $\theta_{1}$ and mass moment of inertia $\theta_{2}$ are introduced:

$$
V:=\frac{v}{l}, \quad E:=\frac{e}{l}, \quad \theta_{1}:=\frac{m l b_{\mathrm{ch}}}{J_{\mathrm{st}}}, \quad \theta_{2}:=\frac{m b_{\mathrm{ch}}^{2}}{J_{\mathrm{st}}}+\frac{J_{\mathrm{ch}}}{J_{\mathrm{st}}} .
$$

To obtain the pseudo force we calculate the virtual power of the steering torque

$$
\delta P=M_{\mathrm{st}} \delta \omega_{\mathrm{st}}-M_{\mathrm{st}} \delta \omega_{\mathrm{ch}}
$$

where $\delta$ denotes the virtual quantities and the angular acceleration of the steering system and the chassis are

$$
\omega_{\mathrm{st}}=\dot{\psi}+\dot{\gamma} \quad \text { and } \quad \omega_{\mathrm{ch}}=\dot{\psi}
$$

Thus, using the definition (5) we obtain the pseudo force

$$
\delta P=M_{\mathrm{st}} \delta \sigma \quad \Rightarrow \quad \Gamma=M_{\mathrm{st}}
$$

When steering an automated vehicle the higher-level controller assigns a desired steering angle $\gamma_{\text {des }}$ and the lower-level controller tries to achieve this by assigning a steering torque acting between the chassis and the steered wheel. In this paper we consider $\gamma_{\text {des }} \equiv 0$ that corresponds to the scenario when the vehicle is moving straight and use the linear proportional-derivative (PD) controller

$$
M_{\mathrm{st}}=-k_{\mathrm{p}} \gamma-k_{\mathrm{d}} \dot{\gamma}
$$

where $k_{\mathrm{p}}$ and $k_{\mathrm{d}}$ are the proportional and differential gains, respectively. Note that for the shopping carts these gains are indeed zero. Let us also define

$$
\omega_{\mathrm{n}}:=\sqrt{\frac{k_{\mathrm{p}}}{J_{\mathrm{st}}}} \text { and } \quad \zeta:=\frac{k_{\mathrm{d}}}{J_{\mathrm{st}}}
$$


that represent the natural angular frequency and the damping factor if the front wheel was lifted form the ground while the chassis was kept fixed. Note that, the damping factor and the angular frequency has the same dimension 1/s. Using (5) and (15) we obtain the pseudo force

$$
\frac{1}{J_{\mathrm{st}}} \Gamma=-\omega_{\mathrm{n}}^{2} \gamma-\zeta \sigma
$$

Now, summarizing (1), (6) (11), and (18) the equation of motion becomes

$$
\begin{aligned}
\dot{\sigma} & =\frac{E\left(E \theta_{2}+\cos \gamma\right) \sin \gamma}{(E-\cos \gamma)\left(E^{2} \theta_{2}+\cos ^{2} \gamma\right)} \sigma^{2}+\frac{V}{E}\left(\frac{E^{2}-1}{\cos \gamma-E}+\frac{E^{3}\left(\theta_{2}-\theta_{1}\right)+\cos \gamma}{E^{2} \theta_{2}+\cos ^{2} \gamma}\right) \sigma \\
& -V^{2} \frac{E \theta_{1} \sin \gamma}{E^{2} \theta_{2}+\cos ^{2} \gamma}-\omega_{\mathrm{n}}^{2} \frac{(E-\cos \gamma)^{2}}{E^{2} \theta_{2}+\cos ^{2} \gamma} \gamma-\zeta \frac{(E-\cos \gamma)^{2}}{E^{2} \theta_{2}+\cos ^{2} \gamma} \sigma, \\
\dot{\gamma} & =\sigma \\
\dot{x} & =V l \cos \psi \\
\dot{y} & =V l \sin \psi \\
\dot{\psi} & =\frac{E \sigma+V \sin \gamma}{\cos \gamma-E} .
\end{aligned}
$$

Note that the last three equations can be decoupled from the first two, since these can be solved without the coordinates $x, y$ and $\psi$. Thus, in the following part of the paper, our investigation requires the analysis of the equations $19 \mathrm{a}$ and $19 \mathrm{~b}$ only.

\section{Stability and bifurcation analysis}

The goal of the controller 16 is to make the steering angle zero (in order to make the vehicle possess multiple stable and unstable equilibria and singularities. In this section, we investigate the equilibria and singularities as the parameters are varied. We also study the linear stability of the trivial equilibrium in detail and, in case of stability loss, we calculate the arising new equilibria and limit cycle oscillations analytically.

\subsection{Equilibria and singularities in phase space}

To find the equilibria we substitute $\sigma \equiv 0, \gamma \equiv \gamma^{*}$ into Eqs. (19a), $19 \mathrm{~b}$ that yields the nonlinear algebraic equation

$$
V^{2} E \theta_{1} \sin \gamma^{*}+\omega_{\mathrm{n}}^{2}\left(E-\cos \gamma^{*}\right)^{2} \gamma^{*}=0,
$$


for $\gamma^{*}$. Although, no closed-form analytic solution exist, the number of equilibria can be determined as a function of the dimensionless system parameters $\theta_{1} V^{2} / \omega_{\mathrm{n}}^{2}$ and $E$ as shown in Fig. 3 . Notice that in the practically important parameter domain $|E|<1$ infinitely many equilibria exist so that different solutions corresponds to how many times the wheel is turned around. In the special case with $\omega_{\mathrm{n}}=0$ (shopping cart), these equilibria are given by $\gamma^{*}=n \pi, n \in \mathbb{Z}$. If the speed $V$ or the caster length $E$ is zero too, then arbitrary $\gamma^{*}$ gives an equilibrium except those that yield singularities (see below). Finally, notice that the trivial equilibrium $\gamma^{*}=0$ exists for all system parameters, but other equilibria may arise in its vicinity due to pitchfork bifurcation (see Sec. 3.3 for more details).

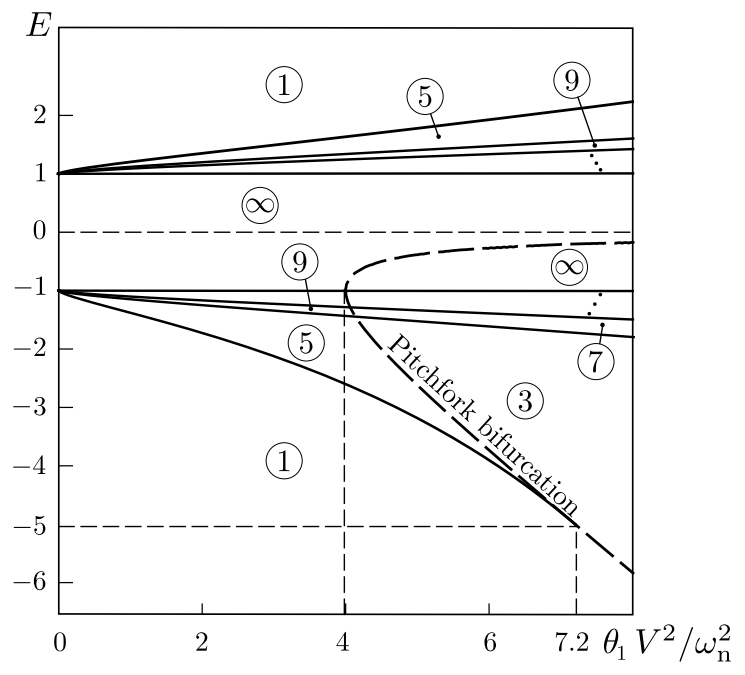

Figure 3: Number of equilibria for different system parameters. Encircled numbers refer to the number of equilibria in the domain.

In nonholonomic systems, kinematic singularity may exist, where the constraints (2)-44 become linearly dependent. This phenomenon occurs generally for wheeled snake models independently of the selection of the configuration coordinates and pseudo velocities, see in [30]. In our system, singularities occur when the right hand side of $19 \mathrm{a}$ approaches infinity. This happens for

$$
\gamma=\tilde{\gamma}= \pm \arccos E+2 n \pi, \quad n \in \mathbb{Z},
$$

when

$$
\sigma \neq 0 \quad \text { and } \quad \sigma \neq \mp \frac{V}{E} \sqrt{1-E^{2}}
$$


see the phase plane sketch in Fig. 4.

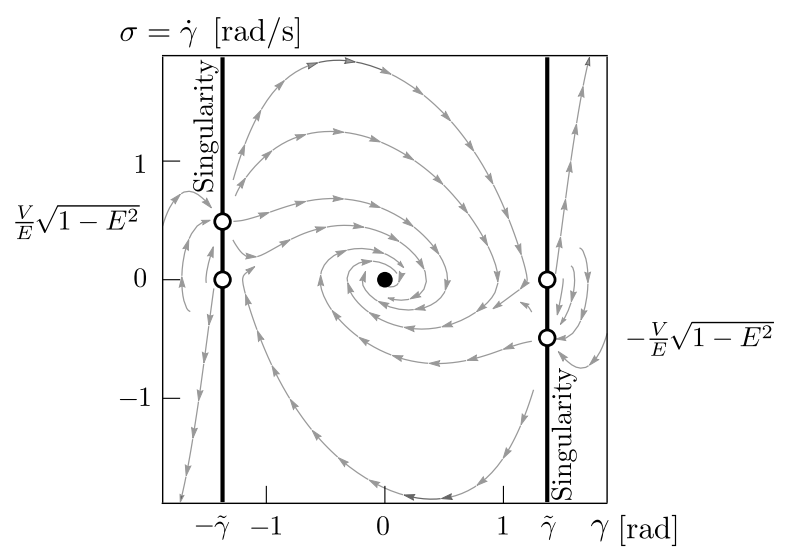

Figure 4: Phase plane sketch constructed for parameters $\theta_{2}=4.5, \theta_{1}=4, \omega_{\mathrm{n}}=1[\mathrm{rad} / \mathrm{s}], \zeta=0.5[\mathrm{rad} / \mathrm{s}]$ and $V=0.1[1 / \mathrm{s}]$. The black dot refers to the equilibrium, black continuous lines indicate singularities 21, while circles denote non-singular points 22 .

\subsection{Linear stability of the rectilinear motion}

As mentioned above, the trivial equilibrium $\gamma \equiv 0$ exist for any system parameter, cf. (20). Linearizing the equation of motion $119 \mathrm{a})(19 \mathrm{~b})$ about the trivial solution yields:

$$
\left[\begin{array}{c}
\dot{\sigma} \\
\dot{\gamma}
\end{array}\right]=\left[\begin{array}{cc}
-b & -c \\
1 & 0
\end{array}\right]\left[\begin{array}{l}
\sigma \\
\gamma
\end{array}\right],
$$

where

$$
\begin{aligned}
& b=\frac{V\left(E^{2} \theta_{1}+E \theta_{2}+1\right)+\zeta(E-1)^{2}}{E^{2} \theta_{2}+1}, \\
& c=\frac{V^{2} E \theta_{1}+\omega_{\mathrm{n}}^{2}(E-1)^{2}}{E^{2} \theta_{2}+1} .
\end{aligned}
$$

By substituting an exponential trial solution into (23) we obtain the characteristic equation

$$
\lambda^{2}+b \lambda+c=0 .
$$

According to the Routh-Hurwitz criteria the corresponding characteristic roots have negative real parts if and only if

$$
b>0 \text { and } c>0 \text {, }
$$


that ensures the asymptotic stability of the trivial equilibrium.

Stability may be lost through a steady state bifurcation when $c=0$ (corresponding to $\lambda=0$ in (25)), that occurs at the critical speed

$$
V=V_{\mathrm{P}}:= \pm \frac{\omega_{\mathrm{n}}(E-1)}{\sqrt{-E \theta_{1}}}
$$

(cf. 24b); see dashed curve in Fig. 3. Notice that such stability loss can only happen for $E<0$. Moreover, $c>0$ corresponds to $V^{2}<V_{\mathrm{P}}^{2}$, that is, stability loss occurs as the magnitude of $V$ bifurcation occurs at this point.

On the other hand, stability may also be lost through a Hopf bifurcation when $b=0$ while $c>0$ (corresponding to $\lambda= \pm \mathrm{i} \omega= \pm \mathrm{i} \sqrt{c}$ in (25)), that happens at the critical speed

$$
V=V_{\mathrm{H}}:=-\frac{\zeta(E-1)^{2}}{E^{2} \theta_{1}+E \theta_{2}+1}
$$

when $V^{2} E \theta_{1}+\omega_{\mathrm{n}}^{2}(E-1)^{2}>0$; cf. 24a and 24b. Notice that 28) is singular at the negative caster lengths

$$
E=E_{\mathrm{H}}^{\mathrm{dis}}:=-\frac{\theta_{2}}{2 \theta_{1}} \pm \sqrt{\left(\frac{\theta_{2}}{2 \theta_{1}}\right)^{2}-\frac{1}{\theta_{1}}}
$$

if $\theta_{2}^{2}>4 \theta_{1}$.

\subsection{Pitchfork bifurcation}

An approximation can be given for the equilibria that arise from steady state bifurcation. The third order Taylor series expansion of 200 around zero yields the equilibria

$$
\gamma^{*} \approx \pm \sqrt{\frac{V^{2} E \theta_{1}+\omega_{\mathrm{n}}^{2}(E-1)^{2}}{V^{2} E \theta_{1} / 6-\omega_{\mathrm{n}}^{2}(E-1)}}= \pm \sqrt{\frac{V^{2}-V_{\mathrm{P}}^{2}}{V^{2} / 6+V_{\mathrm{P}}^{2} /(E-1)}},
$$

which is a good approximation when $V^{2} \approx V_{\mathrm{P}}^{2}$. Notice that when $-5<E<0$, the expression under the square root is positive for $V^{2}<V_{\mathrm{P}}^{2}$ and the solutions 30 coexist with the stable trivial solution $\gamma^{*}=0$. That is, 30 correspond to unstable equilibria and a subcritical pitchfork bifurcation occurs at $V^{2}=V_{\mathrm{P}}^{2}$. Similar, argument can be used to conclude that the bifurcation is supercritical when $E<-5$. The changes in criticality can also be observed in Fig. 3 by looking at the numbers of equilibria on the two sides of the dashed curve (cf. 27)). 
At $V=V_{\mathrm{H}}$ the third degree Taylor expansion of $19 \mathrm{a}, 19 \mathrm{~b}$ yields

$$
\left[\begin{array}{c}
\dot{\sigma} \\
\dot{\gamma}
\end{array}\right]=\left[\begin{array}{cc}
0 & -\omega^{2} \\
1 & 0
\end{array}\right]\left[\begin{array}{l}
\sigma \\
\gamma
\end{array}\right]+\left[\begin{array}{c}
k_{21}^{\mathrm{H}} \sigma^{2} \gamma+k_{12}^{\mathrm{H}} \sigma \gamma^{2}+k_{03}^{\mathrm{H}} \gamma^{3} \\
0
\end{array}\right],
$$

where $\omega=\sqrt{c}(\mathrm{cf} .24 \mathrm{~b})$ ) and

$$
\begin{aligned}
& k_{21}^{\mathrm{H}}=\frac{1}{E^{2} \theta_{2}+1}+\frac{1}{E-1}, \\
& k_{12}^{\mathrm{H}}=\frac{V_{\mathrm{H}}}{2 E}\left(\frac{E+1}{E-1}-\frac{E^{2}\left(2 E\left(\theta_{1}-\theta_{2}\right)+\theta_{2}\right)-1}{\left(E^{2} \theta_{2}+1\right)^{2}}\right)-\zeta \frac{E(E-1)\left(E \theta_{2}+1\right)}{\left(E^{2} \theta_{2}+1\right)^{2}}, \\
& k_{03}^{\mathrm{H}}=E \frac{V_{\mathrm{H}}^{2} \theta_{1}\left(E^{2} \theta_{2}-5\right)-6 \omega_{\mathrm{n}}^{2}(E-1)\left(E \theta_{2}+1\right)}{6\left(E^{2} \theta_{2}+1\right)^{2}} .
\end{aligned}
$$

The eigenvalues and eigenvectors of the coefficient matrix of the linear part in (31) are

$$
\lambda_{1,2}= \pm \mathrm{i} \omega \quad \text { and } \quad \mathbf{s}_{1,2}^{\mathrm{T}}=\left[\begin{array}{ll} 
\pm \mathrm{i} \omega & 1
\end{array}\right] .
$$

To have Hopf bifurcation, the transversality condition

$$
\operatorname{Re} \lambda^{\prime}\left(V_{\mathrm{H}}\right)=-\frac{E^{2} \theta_{1}+E \theta_{2}+1}{2\left(E^{2} \theta_{2}+1\right)} \neq 0
$$

has to be satisfied. Correspondingly, instead of Hopf bifurcation, some other kind of bifurcation exist when $E=E_{\mathrm{H}}^{\text {dis }}$; see in 29.

Let us introduce the new variables:

$$
\left[\begin{array}{l}
\sigma \\
\gamma
\end{array}\right]=\mathbf{T}\left[\begin{array}{l}
\xi \\
\eta
\end{array}\right]=\left[\begin{array}{c}
\omega \eta \\
\xi
\end{array}\right]
$$

where the transformation matrix is given by

$$
\mathbf{T}:=\left[\begin{array}{ll}
\operatorname{Re} \mathbf{s}_{1} & \operatorname{Im} \mathbf{s}_{1}
\end{array}\right]=\left[\begin{array}{ll}
0 & \omega \\
1 & 0
\end{array}\right] .
$$

Accordingly, the Jordan-normal form of 31 can be written as

$$
\left[\begin{array}{c}
\dot{\eta} \\
\dot{\xi}
\end{array}\right]=\left[\begin{array}{cc}
0 & -\omega \\
\omega & 0
\end{array}\right]\left[\begin{array}{l}
\eta \\
\xi
\end{array}\right]+\left[\begin{array}{c}
k_{21}^{\mathrm{H}} \omega \eta^{2} \xi+k_{12}^{\mathrm{H}} \eta \xi^{2}+k_{03}^{\mathrm{H}} \xi^{3} / \omega \\
0
\end{array}\right] .
$$


Then the so-called Poincaré-Lyapunov coefficient

$$
\delta_{\mathrm{H}}=\frac{1}{8} k_{12}^{\mathrm{H}}=-\zeta \frac{(E-1)\left(E\left(2+3 \theta_{2}+E\left(2 \theta_{1}+\theta_{2}\right)\right)+2\right)}{16\left(E^{2} \theta_{2}+1\right)\left(E^{2} \theta_{1}+E \theta_{2}+1\right)}
$$

can be determined via the Bautin formula 31. If $\delta_{\mathrm{H}}<0$, supercritical Hopf bifurcation occurs. That is, when $V_{\mathrm{H}}>0$ stable limit cycle exists for $V>V_{\mathrm{H}}>0$ (where the trivial equilibrium is unstable). Similarly, when $V_{\mathrm{H}}<0$ stable limit cycle exists for $V<V_{\mathrm{H}}$. On the contrary, if $\delta_{\mathrm{H}}>0$ then the Hopf bifurcation is subcritical. In this case, when $V_{\mathrm{H}}>0$ unstable limit cycle arises for $V<V_{\mathrm{H}}$ (where the trivial equilibrium is stable). Similarly, $V_{\mathrm{H}}<0$ unstable limit cycle exists for $V>V_{\mathrm{H}}$.

According to (38), the sense of the Hopf bifurcation changes at

$$
E=1, \quad E=E_{\mathrm{H}}^{ \pm}, \quad \text { and } \quad E=E_{\mathrm{H}}^{\mathrm{dis}},
$$

where $E_{\mathrm{H}}^{\mathrm{dis}}$ is given by 29 and

$$
E_{\mathrm{H}}^{ \pm}=-\frac{2+3 \theta_{2}}{2\left(2 \theta_{1}+\theta_{2}\right)} \pm \frac{\sqrt{\left(2+3 \theta_{2}\right)^{2}-8\left(\theta_{2}+2 \theta_{1}\right)}}{2\left(2 \theta_{1}+\theta_{2}\right)}
$$

It can be seen, that $E_{\mathrm{H}}^{ \pm}<0$ when $\theta_{2}>0$ and $\theta_{1}>0$. Also notice that $E_{\mathrm{H}}^{ \pm}$cannot be interpreted if

$$
\theta_{1}>\frac{1}{4} \quad \text { and } \quad \theta_{2}<\frac{2}{9}\left(-1+2 \sqrt{9 \theta_{1}-2}\right)
$$

so for these values the sense of the Hopf bifurcation does not change.

One can also give an approximation for the amplitude of the arising limit cycle:

$$
\gamma_{\mathrm{r}}=\sqrt{-\frac{\operatorname{Re} \lambda^{\prime}\left(V_{\mathrm{H}}\right)}{\delta_{\mathrm{H}}}\left(V-V_{\mathrm{H}}\right)}=\sqrt{-\frac{8\left(E^{2} \theta_{1}+E \theta_{2}+1\right)^{2}}{\zeta(E-1)\left(E\left(2+3 \theta_{2}+E\left(2 \theta_{1}+\theta_{2}\right)\right)+2\right)}\left(V-V_{\mathrm{H}}\right)},
$$

since the linear transformation in 35 has no effect on $\gamma$ (i.e., $\gamma=\xi$ ).

\section{Application to vehicles}

In this section, we apply the mathematical results presented above to vehicles. First, we discuss the simple case of the shopping cart in order the characterize the behavior when no control is applied. Then we turn our attention to the steering control of automated vehicles. 


\subsection{Shopping cart}

Let us consider $k_{\mathrm{p}}=0, k_{\mathrm{d}}=0$ in (16) that yields $\omega_{\mathrm{n}}=0, \zeta=0$ (cf. 17). The corresponding stability chart is plotted in the $(V, E)$-plane in Fig. $5(a)$. In this case the stability condition 26 yields $V>0$ and $E>0$ independent of $\theta_{1}$ and $\theta_{2}$; cf. 24a and 24b). However, the arising bifurcations are degenerate in nature as the trivial equilibrium is not isolated: an arbitrary $\gamma^{*}$ satisfies (20) when $V=0$ or $E=0$. The former one corresponds to the steady cart while in the later case the caster is missing and the rear point of the cart moves on a circle of constant radius $l / \tan \gamma^{*}$ where $\gamma^{*}$ is given by the initial conditions. Along the line section $V=0, E>0$ we have $b=0$ and $c=0$ yielding a double-zero stability loss instead of a Hopf bifurcation; see 25). Along the line section $E=0, V>0$ we have $b=V$ and $c=0$ that corresponds to a degenerate pitchfork bifurcation.

To clarify what happens when crossing these boundaries in the $(V, E)$-plane, the typical phase portraits in the vicinity of the trivial equilibrium are sketched in Fig. 5(b) corresponding to the different domains of the stability chart in Fig. 5(a). The phase space plots in Fig. 5(c) are generated by numerical simulation and they correspond to the points marked I-IV in Fig. 5(a). These display the global dynamics by showing the vector field as gray and highlighting a particular trajectory as a black curve. To visualize the corresponding motion of the cart in physical space, traces made by the front and rear wheels in the $(X, Y)$-plane are displayed in Fig. 5(d).

Point $I$ is chosen from the linearly stable domain, that is, all trajectories starting in the vicinity of the trivial equilibrium approach the equilibrium as shown in panel (b). The phase space plot in panel (c) show that the trivial equilibrium is globally stable, that is, from any non-singular initial condition the cart approaches a forward rectilinear motion as visualized in panel (d). (Note that there is a $2 \pi$ periodicity in state space, that is, the states at $\gamma=\pi$ and at $\gamma=-\pi$ are equivalent.) In case II the trivial equilibrium is of saddle type as shown in panel (b) and after moving away from it the trajectory in panel (c) passes through a non-singular point before approaching the stable equilibrium corresponding to flipped wheel that is globally stable. For point III the trivial equilibrium (corresponding to a reversing cart) is still of saddle type. After moving away from this equilibrium the trajectory approaches a non-singular point with constant steering angle close to $\pi / 2$. This corresponds to a circular path in the $(X, Y)$-plane as depicted in panel (d). Finally, in case IV the trivial equilibrium is an unstable node, that is, all trajectories move away from it as shown in panel (b). Panel (c) shows a trajectory that goes through a non-singular point and then approaches 


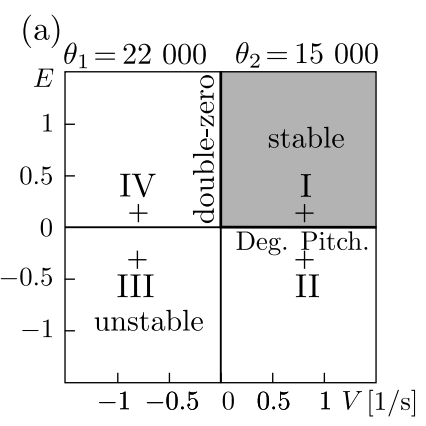

(b) (c)

(d)
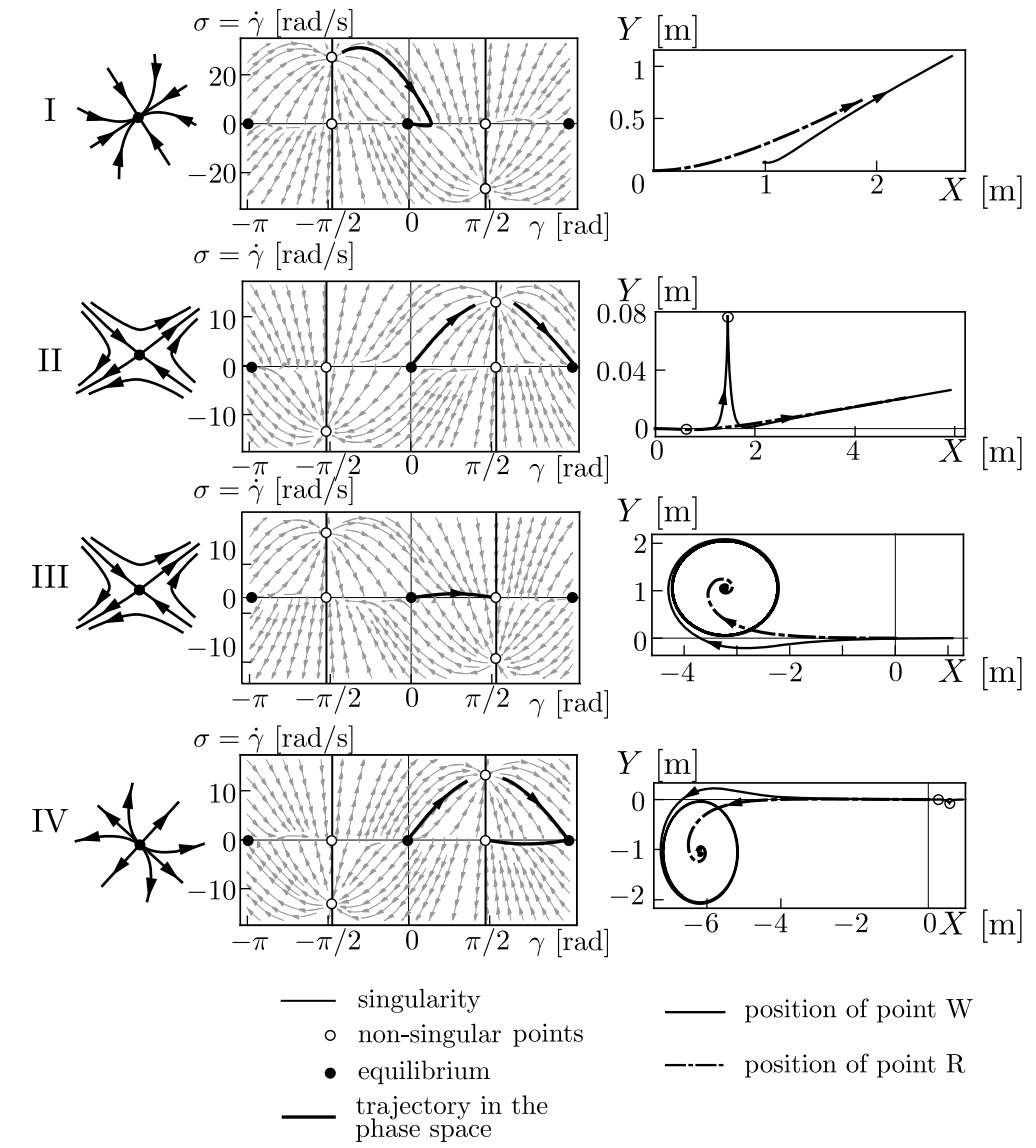

- non-singular points

phase space

Figure 5: (a) Stability chart for the shopping cart with no damping $\left(\omega_{\mathrm{n}}=0, \zeta=0\right)$. (b) The sketch of the flow around the trivial equilibrium for the points marked I-IV in panel (a). (c) Simulation results in the phase plane. (d) Corresponding trajectories in the $(X, Y)$-plane.

the "flipped" equilibrium (that is of saddle type) before reaching another non-singular point with constant steering angle. Thus, the cart again ends up on a circular path in the $(X, Y)$-plane.

We remark that the non-singular points introduce nondeterminism to the system. For example, in case IV the trajectory existing the first non-singular point is on the left side of the stable manifold

of the saddle equilibrium. As a result it eventually ends up at the non-singular point around $(\pi / 2,0)$. However, the trajectory may exit the non-singular point on the right side of the stable manifold. In this case, after "swinging by" the saddle equilibrium it ends up at the non-singular point around $(-\pi / 2,0)$ 
To better understand the dynamics of the trolley example (and to link it to the steering control by the front and rear wheels in the $(X, Y)$-plane are depicted in Fig. 6(e). In case I the trajectory in the damped model in Fig. 6 is qualitatively similar to the trajectory of the undamped model in Fig. 5), that is, the forward motion of the shopping cart is asymptotically stable independent from 


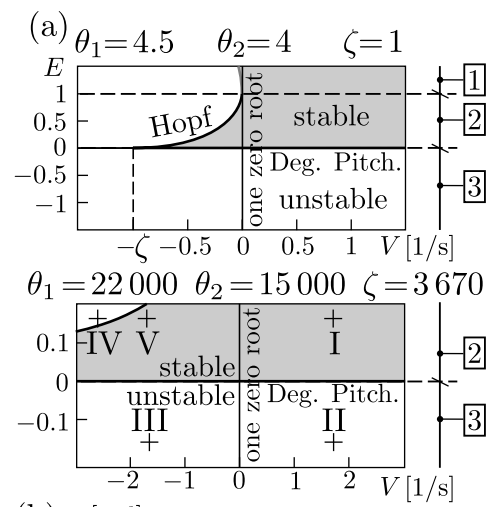

(c)

(d)

(e)
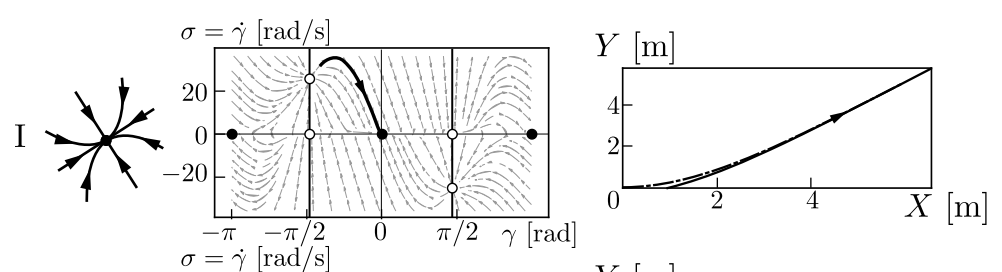

II
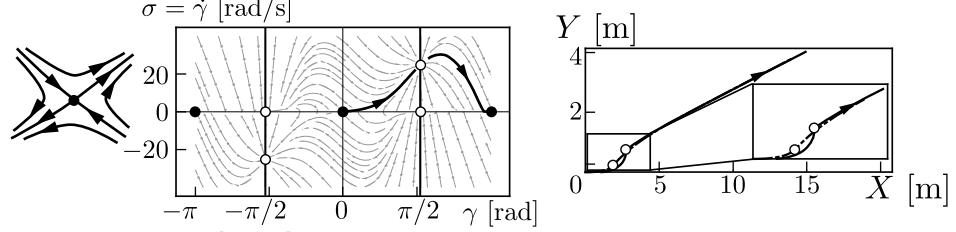

(b) $\gamma[\mathrm{rad}]$
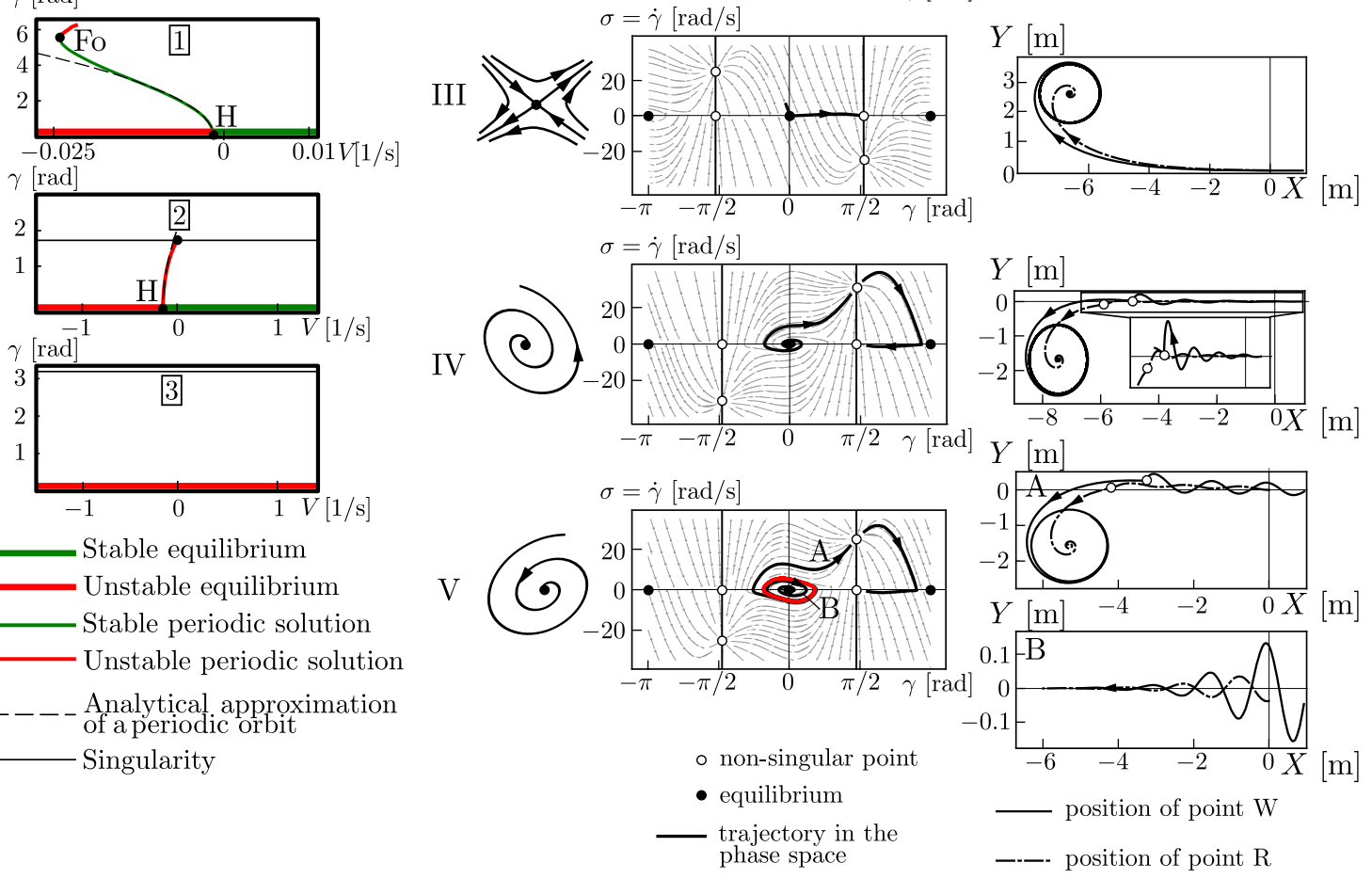

Figure 6: (a) Stability charts for the shopping cart with non-zero damping $\left(\omega_{\mathrm{n}}=0, \zeta \neq 0\right)$. (b) Bifurcation diagrams corresponding to the horizontal cross sections of panel (a). (c) (b) The sketch of the flow around the trivial equilibrium for the points marked I-V in panel (a). (d) Simulation results in the phase plane. (e) Corresponding trajectories in the $(X, Y)$-plane.

the damping. Also, in cases II and III, the motions corresponding to the points II and III for the damped model are similar to those for the undamped model. Qualitative differences between the undamped and damped cases can be observed when comparing the results for points IV and V in 
Fig. 6 to those for point IV in Fig. 5 . When the swiveling wheel is damped the stability of the rectilinear motion is lost via Hopf bifurcation. Thus, in the unstable case IV oscillations develop before the trajectory passes through a non-singular point. Then, similar to the undamped case, the system "swings by" a saddle state before approaching another non-singular state corresponding to circular motion of the cart. However, for smaller reversing speed the rectilinear motion can remain stable. Case V B shows a scenario when small disturbances (corresponding to initial condition within the red unstable limit cycle) die out while case V A demonstrates that larger disturbances still grow leading to as similar global behavior as in case IV.

\subsection{Automated vehicles}

When designing the steering control of vehicles our goal is to select the control gains $k_{\mathrm{p}}$ and $k_{\mathrm{d}}$ in (16) such that the rectilinear motion is asymptotically stable (at least at the linear level). Thus, in this sections we study the cases when both gains are positive corresponding to positive $\omega_{\mathrm{n}}$ and $\zeta$ parameters (cf. 17). In this case the stability boundaries are described by $V=V_{\mathrm{P}}$ (see (27)) and $V=V_{\mathrm{H}}$ (see (28)), corresponding to pitchfork and Hopf bifurcations, respectively.

The stability charts are plotted in Fig. 7(a) in the $\left(V / \omega_{\mathrm{n}}, E\right)$-plane for different values of $\zeta / \omega_{\mathrm{n}}, \theta_{1}$ and $\theta_{2}$ so that all topologically different cases are represented. Indeed, the dashed pitchfork curves correspond to the dashed curve in Fig. 3 and recall that for $-5<E<0$ the pitchfork bifurcation is always subcritical; see 30 . Notice that the Hopf curves may have discontinuities corresponding to the singularities at $E=E_{\mathrm{H}}^{\text {dis }}$; cf. 29 . Again, the criticality of the Hopf bifurcation is determined by 38 and sub- and supercritical bifurcations are distinguished by black and gray curves.

In Fig. 7 (b), the bifurcation diagrams are plotted with respect to the dimensionless speed $V / \omega_{\mathrm{n}}$ as bifurcation parameter for fixed values of the dimensionless caster length $E$ as highlighted by framed numbers in the stability charts. Dashed black curves correspond to the analytical approximations (30) and 42 while the colored curves are generated by numerical continuation and the same notation is used as in Fig. 6. Apart from Hopf $(\mathrm{H})$, pitchfork $(\mathrm{P})$, and fold (Fo) bifurcations we also found heteroclinic (He) bifurcation when a limit cycle collides with a nontrivial equilibrium. Recall that for road vehicles and harvesters we have $|E|<<1$ as the caster length is much smaller than the wheelbase, cf. (12). Thus, here we only describe the bifurcation diagrams $2,3,4,5,6$ in Fig. 7(b) in detail.

Bifurcation diagram 2 (valid for $0<E<1$ ) belongs to the car model according to Fig. 2. The 

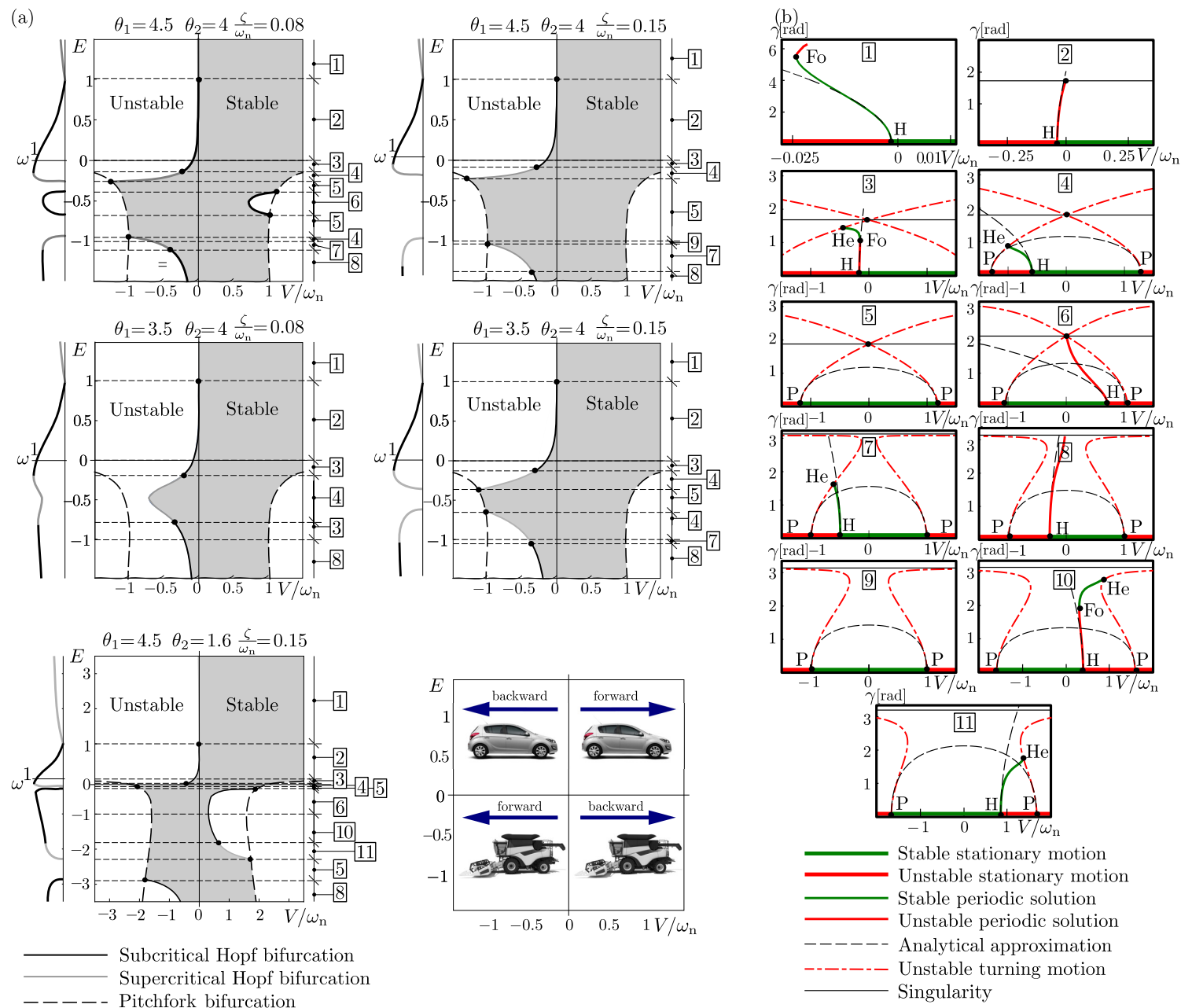

Figure 7: Stability charts and bifurcation diagrams for different parameters $\zeta / \omega_{\mathrm{n}}, \theta_{1}$ and $\theta_{2}$ as indicated. (a) Linear stability charts, where the sense of the Hopf bifurcation is distinguished by black (subcritical) and gray (supercritical). The angular frequency of the arising periodic solution along the Hopf bifurcation curve is also plotted on the left sides of the stability charts. (b) Bifurcation diagrams, where $\mathrm{H}$ and $\mathrm{P}$ refer to Hopf and pitchfork bifurcation of the trivial solution, Fo denotes the fold bifurcation of periodic orbits and He represents heteroclinic bifurcation.

stability of the forward rectilinear motion of an automated vehicle is ensured by the steering system but when reversing motion becomes linearly unstable for large enough reverse speed. Moreover, an unstable limit cycle arises even when the reverse motion is linearly stable, that is, large enough perturbations still give rise to oscillations. In order to illustrate this behavior the stability chart and the bifurcation diagram are reproduced while varying the vehicle speed $v$ and the caster length 
$e$ in Fig. 8 for some realistic vehicle parameters listed in the caption. We also show the results of numerical simulations for the points marked I-III on the stability chart. Case I shows that the forward rectilinear motion is stable while case III shows that reverse motion is unstable if the speed is high enough. Case II corresponds to the reverse motion with lower speed. In this case choosing initial condition B the oscillations decay and the vehicle approaches a stable rectilinear motion. On the other hand for initial condition A oscillations with growing amplitude appear.
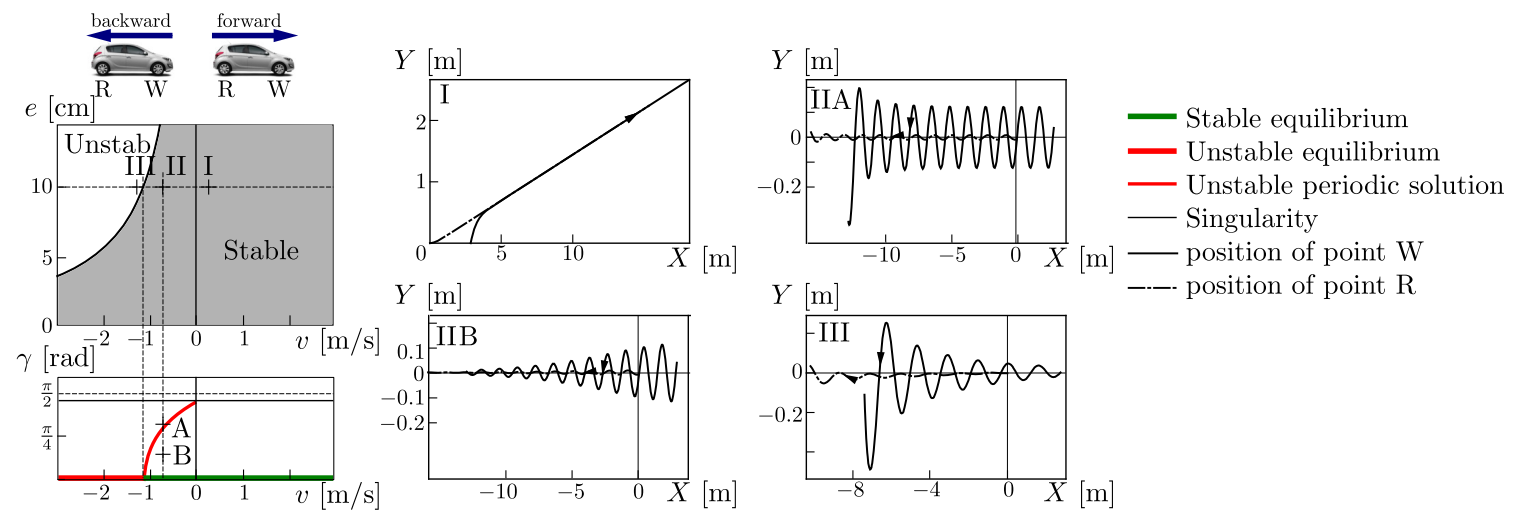

Figure 8: Stability chart, bifurcation diagrams, and numerical simulations for $m=1600 \mathrm{~kg}, J_{\mathrm{ch}}=2800 \mathrm{kgm}^{2}$, $l=2.85 \mathrm{~m}, b_{\mathrm{ch}}=1.5 \mathrm{~m}, J_{\mathrm{st}}=10 \mathrm{kgm}^{2}, e=0.1 \mathrm{~m}, k_{\mathrm{p}}=400 \mathrm{Nm}$ and $k_{\mathrm{d}}=100 \mathrm{Nms}$.

Based on the bifurcations diagrams 3, 4, 5, 6 in Fig.7(b) (for $E<0$ ), neither the forward nor the reverse motion of harvesters are stable for large speed justifying the strict speed limit regulations for these vehicles. In the bifurcation diagrams 3 and 4 , there are limit cycles at negative speed (forward motion here) corresponding to wheel shimmy. In bifurcation diagram 3, a subcritical Hopf bifurcation occurs: although the straight running motion is linearly stable, the wheel may start to oscillate when sufficiently large perturbations are applied. Moreover, if the speed of the harvester is only slightly higher then the linear stability limit, large amplitude oscillations appear. On the contrary, the bifurcation diagram 4 predicts small amplitude vibrations for larger caster lengths due to the existence of a supercritical Hopf bifurcation.

In Fig. 9 the stability chart is reproduced for some realistic vehicle parameters (see caption) while varying the vehicle speed $v$ and the caster length $e$ and the simulation results are presented for the points marked I-V. Note that in cases I and II the transient are not shown. Cases I and II demonstrate a stability loss via subcritical Hopf bifurcation. In case I, even though the rectilinear 


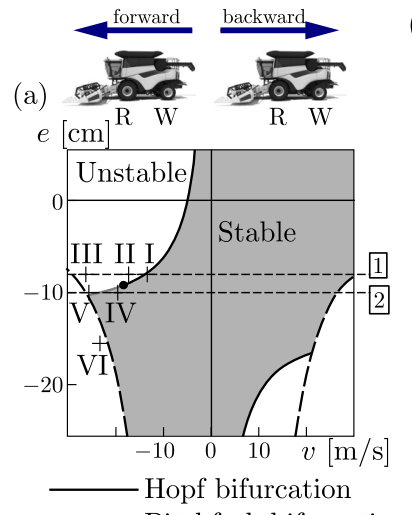

(c) (b)
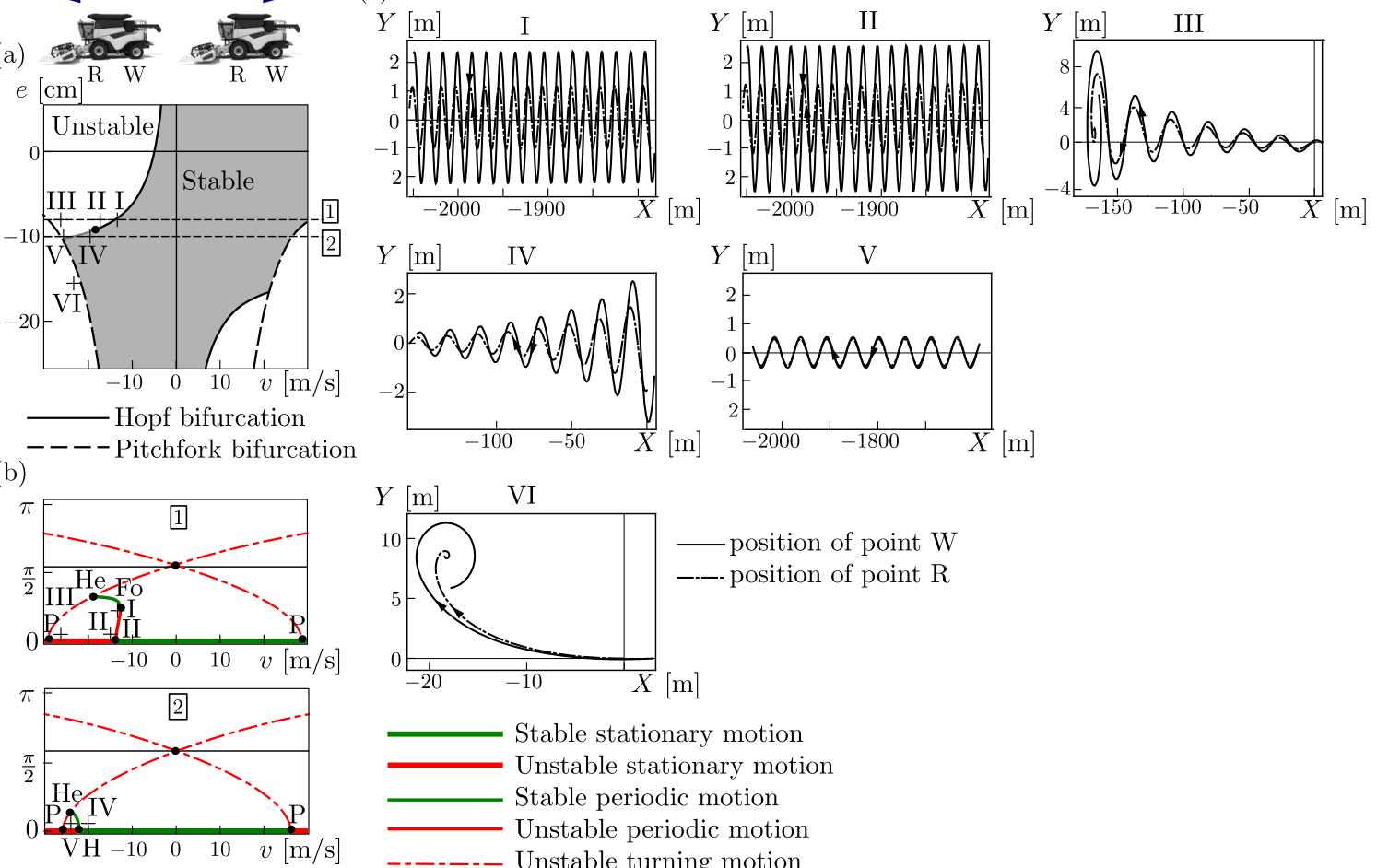

Stable stationary motion

Unstable stationary motion

Stable periodic motion

Unstable periodic motion
----- Unstable turning motion

Singularity

Figure 9: Stability chart, bifurcation diagram, and numerical simulations for $m=17400 \mathrm{~kg}, J_{\mathrm{ch}}=24500 \mathrm{kgm}^{2}$, $l=5 \mathrm{~m}, b_{\mathrm{ch}}=1.8 \mathrm{~m}, J_{\mathrm{st}}=2000 \mathrm{kgm}^{2}, k_{\mathrm{p}}=2000 \mathrm{Nm}$ and $k_{\mathrm{d}}=1000 \mathrm{Nms}$.

motion is linearly stable, oscillations still develop for certain initial conditions. Moreover, when the rectilinear motion becomes unstable, as in case II, large amplitude oscillations also arise. Case III demonstrates that without the presence of large amplitude oscillations the cart reaches circular motion. Cases IV and V demonstrate a stability loss via supercritical Hopf bifurcation. In case IV the rectilinear motion is asymptotically stable while in case $\mathrm{V}$ it is unstable and small amplitude vibrations develop. Finally, case VI demonstrates a stability loss via pitchfork bifurcation leading to a turning motion with constant steering angle.

\section{Conclusion}

In this article we presented a low degree-of-freedom vehicle model that allowed us to study the lateral and yaw dynamics of shopping cars, automobiles, harvesters. We investigated the nonlinear 
dynamics using analytical and numerical bifurcation analysis while applying a PD controller that intented to stabilize the rectilinear motion. By analyzing the linear stability of equilibria we found that the vehicle may lose stability in oscillatory and non-oscillatory ways corresponding to Hopf and pitchfork bifurcations, respectively. These led to a large variety of qualitatively different behaviors that were visualized using stability charts, bifurcation diagrams, phase plane plots and wheel traces. We applied the theoretical results for different vehicle classes.

For the (American) shopping cart we proved that without damping the forward rectilinear motion is globally stable while the reverse rectilinear motion is unstable. We also showed that adding damping can stabilize the reverse motion for low speed. However, only local stability can be achieved and large perturbations still drive the system away from the equilibrium. We illustrated that the dynamics of automobiles is qualitatively similar to those of the damped shopping carts. When moving forward stability can be easily maintained even in the presence of large perturbations, but stability of the reverse motion can only be ensured up to about 2 meters per seconds and only small disturbances can be tolerated. In contrarily, harvesters can maintain stability while moving forward or backward up about 20 meters per second but dangerous oscillations appear for higher speed. Our results imply that the front wheel steering is has the advantage in terms of stability (when moving forward) while rear wheel steering provides better maneuverability.

Indeed, our results only hold for the (linear) PD controller applied in the paper and they do not exclude the possibility of having nonlinear controllers of increased performance. One may use Lyapunov based techniques in order to design such controllers while barrier functions may be applied in order to guarantee safety. Combining such lower-level controllers with higher-level motion planning is another interesting future research direction.

\section{Acknowledgment}

This research was partly supported by the National Research, Development and Innovation Office under grant no. NKFI-128422 and by the Higher Education Excellence Program of the Ministry of Human Capacities in the frame of Artificial intelligence research area of Budapest University of Technology and Economics (BME FIKP-MI). 


\section{References}

${ }_{300}$ [1] R. Marino, S. Scalzi, M. Netto, Nested PID steering control for lane keeping in autonomous vehicles, Control Engineering Practice 19 (12) (2011) 1459-1467.

[2] P. Falcone, F. Borrelli, J. Asgari, H. E. Tseng, D. Hrovat, Predictive active steering control for autonomous vehicle systems, IEEE Transactions on Control Systems Technology 15 (3) (2007) $566-580$.

[3] G. V. Raffo, G. K. Gomes, J. E. Normey-rico, C. R. Kelber, L. B. Becker, A predictive controller for autonomous vehicle path tracking, IEEE Transactions on Intelligent Transportation Systems 10 (1) (2009) 92-102.

[4] M. Jalali, A. Khajepour, S. Chen, B. Litkouhi, Handling delays in yaw rate control of electric vehicles using model predictive control with experimental verification, Journal of Dynamic Systems, Measurement, and Control 139 (12) (2017) 121001.

[5] K. Kritayakirana, J. C. Gerdes, Controlling an autonomous racing vehicle: using feedforward and feedback to control steering and speed, in: Proceedings of the ASME Dynamic Systems and Control Conference, 2009, pp. 173-180, paper no. DSCC2009-2572.

[6] X. Li, Z. Sun, D. Liu, Q. Zhu, Z. Huang, Combining local trajectory planning and tracking control for autonomous ground vehicles navigating along a reference path, 17th International IEEE Conference on Intelligent Transportation Systems (2014) 725-731.

[7] H. B. Pacejka, Tyre and vehicle dynamics, 2nd Edition, Butterworth-Heinemann, Oxford, UK, 2006.

[8] D. Takács, G. Stépán, Contact patch memory of tyres leading to lateral vibrations of four-wheeled vehicles, Philosophical Transactions of the Royal Society A 371 (1993) (2013) 20120427.

[9] P. Appell, Sur l'intégration des équations du mouvement d'un corps pesant de révolution roulant par une arête circulaire sur un plan horizontal; cas particulier du cerceau, Rendiconti del circolo matematico di Palermo 14 (1900) 1-6. 
[10] T. R. Kane, D. A. Levinson, Dynamics, theory and applications, Internet-First University Press., Ithaca, NY, USA, 2005.

[11] A. M. Bloch, P. E. Crouch, Newton's law and integrability of nonholonomic systems, SIAM Journal on Control and Optimization 36 (6) (1998) 2020-2039.

[12] A. Ruina, Non-holonomic stability aspects of piecewise-holonomic systems, Reports on Mathematical Physics 42 (1-2) (1998) 91-100.

[13] A. M. Bloch, Nonholonomic Mechanics and Control, 2nd Edition, Springer, 2015.

[14] M. Hubbard, Lateral dynamics and stability of the skateboard, Journal of Applied Mechanics 46 (1979) 931-936.

[15] B. Várszegi, D. Takács, G. Stépán, S. J. Hogan, Stabilizing skateboard speed-wobble with reflex delay, Journal of The Royal Society Interface 13 (121).

[16] J. P. Meijaard, J. M. Papadopoulos, A. Ruina, A. L. Schwab, Linearized dynamics equations for the balance and steer of a bicycle: a benchmark and review, Proceedings of the Royal Society A 463 (2084) (2007) 1955-1982.

[17] J. D. G. Kooijman, J. P. Meijaard, J. M. Papadopoulos, A. Ruina, A. L. Schwab, A bicycle can be self-stable without gyroscopic or caster effects, Science 332 (6027) (2011) 339-342.

[18] H. B. Pacejka, Non-linearities in road vehicle dynamics, Vehicle System Dynamics 15 (5) (1986) $237-254$.

[19] G. Stépán, Chaotic motion of wheels, Vehicle System Dynamics 20 (6) (1991) 341-351.

[20] D. Takács, G. Stépán, S. J. Hogan, Bifurcations of a towed rigid wheel, in: Proceedings of the 6th European Solid Mechanics Conference, 2006.

[21] N. Tomiati, A. Colombo, G. Magnani, A nonlinear model of bicycle shimmy, Vehicle System Dynamics (2018) 1-21doi:10.1080/00423114.2018.1465574.

[22] Z. Liu, G. Payre, P. Bourassa, Nonlinear oscillations and chaotic motions in a road vehicle system with driver steering control, Nonlinear Dynamics 9 (3) (1996) 281-304. 
[34] J. Sieber, K. Engelborghs, T. Luzyanina, G. Samaey, D. Roose, Dde-biftool manual-bifurcation analysis of delay differential equations, arXiv:1406.7144. 